\title{
Chinese version of the Edinburgh Postnatal Depression Scale was useful for detecting postnatal depression
}

\author{
Lee DT, Yip SK, Chiu HF, et al. Detecting postnatal depression in Chinese women. Validation of the Chinese version of the \\ Edinburgh Postnatal Depression Scale. Br J Psychiatry 1998 May;172:433-7.
}

\section{Question}

What is the usefulness of the Chinese version of the Edinburgh Postnatal Depression Scale (EPDS) in screening for postnatal depression?

\section{Design}

Blinded comparison of scores on depression scales with results of standardised diagnostic interview.

\section{Setting}

Postnatal wards and outpatient clinic of a university affiliated general hospital in Hong Kong.

\section{Patients}

220 women (mean age 29 y) who were admitted to the postnatal wards between November 1996 and January 1997. Women who were not Chinese or did not have permanent residency rights in Hong Kong were excluded. 145 women $(66 \%)$ returned for the 6 week postpartum assessment.

\section{Description of test and diagnostic standard}

Within 2 days of confinement, women completed the EPDS, the Chinese version of the General Health Questionnaire (GHQ), and the 21 item Beck Depression Inventory (BDI). At 6 weeks postpartum, women repeated the EPDS, GHQ, and BDI and also were assessed with the non-patient version of the Structured Clinical Interview for DSM-III-R (SCID-NP) (diagnostic standard) by an investigator who was blinded to the results of the previous assessments.

\section{Main outcome measures}

Sensitivity, specificity, and positive and negative predictive values of the EPDS at various cut points.

\section{Main results}

Of the 145 women who were assessed by the SCID-NP, 8 women $(5.5 \%)$ had major depression and $9(6 \%)$ had minor depression. Using the conventional cut point of $12 / 13$ for diagnosing depression, the EPDS had a sensitivity of $41 \%$ and a specificity of $95 \%$. A receiver operating characteristic (ROC) curve identified $9 / 10$ as the optimal cut point in this Chinese sample (table). The EPDS moderately correlated with the GHQ (Spearman correlation $0.50, \mathrm{p}<0.001)$ and strongly correlated with the BDI (Spearman correlation 0.73, $\mathrm{p}<0.001$ ).

\section{Conclusion}

The Chinese version of the Edinburgh Postnatal Depression Scale had satisfactory sensitivity and specificity using a cut point of $9 / 10$ for detecting depression in Chinese women at 6 weeks postpartum.

Edinburgh Postnatal Depression Scale (EPDS) for detecting 6 week postpartum depression

\begin{tabular}{lllllll}
\hline Cut point & $\begin{array}{l}\text { Sensitivity } \\
(95 \% \text { CI) }\end{array}$ & Specificity (CI) & $+P V^{*}$ & $-P V^{*}$ & $+L R^{*}$ & $-L R^{*}$ \\
\hline $9 / 10$ & $82 \%(57$ to 96$)$ & $86 \%(78$ to 91$)$ & $44 \%$ & $97 \%$ & 5.9 & 0.21 \\
\hline
\end{tabular}

$*+\mathrm{PV}=$ positive predictive value; $-\mathrm{PV}=$ negative predictive value; $+\mathrm{LR}=$ positive likelihood ratio; $-\mathrm{LR}=$ negative likelihood ratio; $\mathrm{CI}$ calculated from data supplied by author; + LR and - LR calculated from data in article.

Source of funding: Hospital Services Research Fund.

For correspondence: Dr D T Lee, Department of Psychiatry, The Chinese University of Hong Kong, Shatin NT, Hong Kong. Fax +852 26377884.

\section{Commentary}

Lee $e t$ al provide a reasonably thorough assessment of the usefulness of a translated Chinese version of the EPDS as a screening instrument for depression in postnatal Chinese women in Hong Kong. Their use of a ROC curve to identify the best cut point is an improvement over many cross cultural studies that simply apply Western cut points. The much lower cut point for the Chinese version indicates that it would not be appropriate to directly compare mean scores on the EPDS between Western and Chinese patient samples.

The authors do not report on the ability of the EPDS to detect changes in the clinical status of patients, so it is not known if this instrument would be useful as a tool to monitor Chinese women treated for postpartum depression. The high correlation of EPDS scores with BDI scores (0.73) suggests that there may, in fact, be no need for a separate scale to assess postpartum depression.

The best study on the population prevalence of mental illnesses done in mainland China using the Present State Examination (PSE) also found extremely low rates of depression (a combined point prevalence of $1.4 \%$ for all affective disorders and neurasthenia), ${ }^{1}$ but such results should to be viewed with caution. There is no evidence of the validity of Western "gold standard" diagnostic measures of depression (SCID-NP or PSE) in Chinese patients, who may experience and report depressive symptoms differently than Western patients. In this study, only 145 of the 330 eligible patients (44\%) were interviewed at 6 weeks postpartum, so the reported prevalence of postpartum depression $(5.5 \%)$ may be an underestimation. Furthermore, the postpartum experience of Hong Kong women may be different from that of women in the rest of mainland China, where the 1 child per family policy is strictly enforced in urban areas and where rural women who deliver female infants are often criticised by family members, so these results cannot be considered representative of all Chinese women.

Michael R Phillips, MD, MA, MPH Research Centre of Clinical Epidemiology Beijing Hui Long Guan Hospital Beijing, People's Republic of China

1 Cooper JE, Sartorius N, editors. Mental disorders in China: results of the national epidemiological survey in 12 areas. London: Gaskell, 1996. 